\title{
Chiral Amidophosphane-Rhodium(I)-Catalyzed Asymmetric Conjugate Arylation of Acyclic Enones with Arylboronic Acids
}

\author{
Qian Chen, ${ }^{a}$ Masami Kuriyama, ${ }^{b}$ Xinyu Hao, ${ }^{b}$ Takahiro Soeta, ${ }^{b}$ Yasutomo Yamamoto, ${ }^{b}$ \\ Ken-ichi YAMADA, ${ }^{b}$ and Kiyoshi TOMIOKA ${ }^{*}, b$ \\ ${ }^{a}$ The Academy of Fundamental and Interdisciplinary Science, Harbin Institute of Technology; Harbin, Heilongjiang \\ 150080, P. R. China: and ${ }^{b}$ Graduate School of Pharmaceutical Sciences, Kyoto University; Yoshida, Sakyo-ku, Kyoto \\ 606-8501, Japan. Received June 5, 2009; accepted June 27, 2009; published online June 30, 2009
}

\begin{abstract}
A catalytic asymmetric conjugate arylation of acyclic $\alpha, \beta$-unsaturated ketones with arylboronic acids was catalyzed by $3 \mathrm{~mol} \%$ of chiral amidomonophosphane 1-rhodium(I) in the presence of potassium hydroxide in a mixture of 1,4-dioxane and water at $70^{\circ} \mathrm{C}$ to afford 1,4-conjugate arylated acyclic ketones with high enantiomeric excess in high chemical yield. Thirteen examples of the reaction demonstrate the general applicability of the catalytic system.
\end{abstract}

Key words catalytic asymmetric reaction; arylation; arylboronic acid; conjugate addition; $\alpha, \beta$-unsaturated ketone

The carbon-carbon bond forming catalytic asymmetric conjugate addition reaction of $\alpha, \beta$-unsaturated carbonyl compounds with organometallic reagents has been the significant milestone of catalytic asymmetric reactions. ${ }^{1-5}$ ) Tremendous efforts on the discovery of efficient chiral sources for the reaction with carbon- and hetero-nucleophiles provided a fruit of chiral sources like phosphorous compounds. ${ }^{6}$ A touchstone for the evaluation of chiral sources has been the asymmetric reaction of cyclohexenone $\mathbf{2}$ with diorganozinc-copper(I), ${ }^{7,8)}$ Grignard reagent-copper(I $)^{9,10)}$ or arylboronic acid-rhodium $(\mathrm{I})^{11-18)}$ reagent-catalyst combinations. We have also engaged in this fascinating challenge and fortunately succeeded in the development of chiral amidophosphane 1-based catalytic asymmetric conjugate addition reaction of $\mathbf{2}$ with organometallic reagents, especially, Grignard reagents ${ }^{19-21)}$ and diorganozincs. ${ }^{2-26)}$ Especially, 1-rhodium(I) catalyzed asymmetric conjugate arylation of $\mathbf{2}$ with arylboronic acids gave $\mathbf{3}$ with excellently high ee of up to $99 \%$ (Chart 1). ${ }^{27-30}$ ) However, there has been known the presence of a large gap in reaction efficiency between the arylation of cyclic and acyclic enones probably due to $s$-trans and $s$-cis conformation differences of enones. Now we describe a catalytic asymmetric arylation of acyclic enones 7 with arylboronic acids $\mathbf{5}$ yielding arylation products $\mathbf{8}$ with relatively high ee.

Acyclic Enones as Good Acceptors for Conjugate Phenylation At the beginning of this arylation study 4methylbenzylidenemalonate $4^{31,32)}$ was examined with its acceptor ability by treating with phenylboronic acid (5a) in a 10:1 mixture of 1,4-dioxane and water under the catalysis of 1-rhodium(I). Unfortunately, the starting 4 was recovered almost quantitatively without detectable production of adduct 6 (Chart 2). This disaster was overcome by the selection of enone 7 as an acceptor for the conjugate arylation.

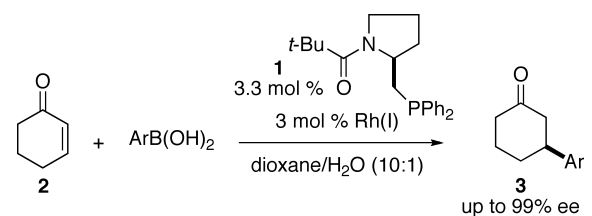

Non-3-ene-2-one (7a) was then selected as the second acceptor because of the simple methyl ketone structure (Table 1). The phenylation of $7 \mathbf{a}$ with 5 eq of phenylboronic acid (5a) was catalyzed by $3 \mathrm{~mol} \%$ of $\mathbf{1}$-rhodium(I) in a $10: 1$ mixture of 1,4-dioxane and water. ${ }^{27)}$ Although the chemical yield was not high under the catalysis of 1-acetylacetonatobis(diethylene)rhodium(I), the desired ketone $\mathbf{8} \mathbf{a a}^{12,33)}$ with $56 \%$ ee was obtained in $35 \%$ isolated yield (Table 1 , entry 1 ). Enantioselectivity (ee\%) was determined by HPLC with chiral stationary phase (Daicel Chiralcel OB-H, hexane/2propanol $=100 / 1,0.5 \mathrm{ml} / \mathrm{min}, 254 \mathrm{~nm}$ ) as described in Experimental. Other two $N$-Boc-valine-connected amidophosphanes 9 and $\mathbf{1 0}^{34}$ (Fig. 1) exhibited the same level of performance to give 8aa with $65 \%$ and $61 \%$ ees, respectively (entries 2, 3). The sense of asymmetric induction was controlled by the pyrrolidine part of the ligands and not by the stereochemistry of the valine moiety; 8aa of the same ab-

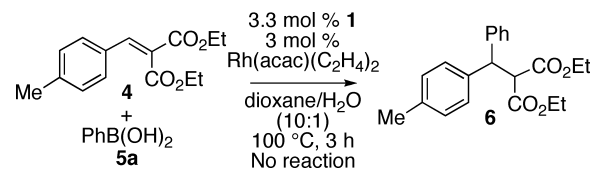

Chart 2. Attempted Arylation of 4-Methylbenzylidenemalonate 4

Table 1. Catalytic Asymmetric Conjugate Phenylation of 7a

\begin{tabular}{|c|c|c|c|c|c|c|c|}
\hline \multirow[b]{2}{*}{ Entry } & \multirow[b]{2}{*}{ Ligand } & \multicolumn{4}{|c|}{$\underset{5 a}{\mathbb{P a}}+\underset{\text { dioxane } / \mathrm{H}_{2} \mathrm{O}(10: 1)}{1.5 \mathrm{~mol} \%\left[\mathrm{RhX}\left(\mathrm{C}_{2} \mathrm{H}_{4}\right)_{2}\right]_{2}}$} & Baa & \multirow[b]{2}{*}{$\begin{array}{c}\text { ee } \\
(\%)\end{array}$} \\
\hline & & $\mathrm{X}$ & Base & $\begin{array}{c}\text { Temp. } \\
\left({ }^{\circ} \mathrm{C}\right)\end{array}$ & $\begin{array}{c}\text { Time } \\
\text { (h) }\end{array}$ & $\begin{array}{c}\text { Yield } \\
(\%)\end{array}$ & \\
\hline 1 & 1 & acac & none & 100 & 3 & 35 & 56 \\
\hline 2 & 9 & acac & none & 100 & 1 & 58 & 65 \\
\hline 3 & 10 & acac & none & 100 & 1 & 61 & 61 \\
\hline 4 & 1 & $\mathrm{Cl}$ & $\mathrm{KOH}$ & 100 & 7 & 96 & 76 \\
\hline 5 & 1 & $\mathrm{Cl}$ & $\mathrm{K}_{3} \mathrm{PO}_{4}$ & 100 & 15 & 90 & 72 \\
\hline 6 & 1 & $\mathrm{Cl}$ & $\mathrm{Et}_{3} \mathrm{~N}^{4}$ & 100 & 15 & 70 & 75 \\
\hline 7 & 9 & $\mathrm{Cl}$ & $\mathrm{KOH}$ & 100 & 31 & 82 & 63 \\
\hline 8 & 1 & $\mathrm{Cl}$ & $\mathrm{KOH}$ & 70 & 20 & 90 & 81 \\
\hline 9 & 1 & $\mathrm{Cl}$ & $\mathrm{KOH}$ & 50 & 23 & 37 & 67 \\
\hline
\end{tabular}

Chart 1. Catalytic Asymmetric Conjugate Arylation of Cyclohexenone 
solute configuration was obtained in the similar level of enantioselectivity.

The reaction was much more improved by using 1-chlorobis(ethylene)rhodium(I) complex in the presence of potassium hydroxide at $100{ }^{\circ} \mathrm{C}$ for $7 \mathrm{~h}$ to give 8aa with $76 \%$ ee in $96 \%$ yield (entry 4 ). Other bases, potassium phosphate and triethylamine, also gave the similar level of efficiency (entries 5, 6). $N$-Boc-L-valine-connected amidophosphane 9 was not a better ligand than 1 to give 8aa with 63\% ee (entry 7). Best efficiency was obtained at $70{ }^{\circ} \mathrm{C}$ reaction temperature for $20 \mathrm{~h}$ to give 8aa with $81 \%$ ee in $90 \%$ yield (entries 8, 9).

Generality in Catalytic Asymmetric Conjugate Arylation The established conditions above were applied to the catalytic asymmetric conjugate arylation of six acyclic enones $7 \mathbf{a}-\mathbf{f}$ with five arylboronic acids $\mathbf{5 a}-\mathbf{e}$ under the catalysis of 1-rhodium(I) in the presence of one equiv of potassium hydroxide in a 10:1 mixture of 1,4-dioxane and water at $70^{\circ} \mathrm{C}$ as has been summarized in Table 2 .

The similar level of relatively high enantioselectivity, $76 \%$ and $78 \%$ ees, were obtained with the use of substituted phenylboronic acids bearing electron-donating methoxy and withdrawing phenyl groups (Table 2, entries 1,2). Relatively bulky isopropyl substituent at the reaction site of enone $\mathbf{7 b}$ did not disturb the reaction to give the arylation products with 76-86\% ee (entries 3-6). A phenyl group bearing an electron-withdrawing trifluoromethyl substituent was introduced in the best enantioselectivity of $86 \%$ (entry 5). The phenyl group at the reaction site of $7 \mathbf{c}$ was deleterious to the enantioselectivity to give arylation products with $65 \%$ and $60 \%$ ees (entries 7, 8). Although ethylketone 7d was usable as an acceptor with $72 \%$ and $74 \%$ enantioselectivity, phenylketones 7e and $\mathbf{7 f}$ were the worst acceptors giving $51 \%$ selectivity (entries 9,10 ). The sense of asymmetric induction was determined as indicated by the comparison of the sign of the specific rotation of $\mathbf{8 d a}$ and $\mathbf{8 f a}$ with those re-

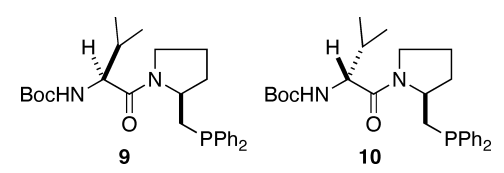

Fig. 1. $N$-Boc-L- and D-Valine Connected Amidophosphanes $\mathbf{9}$ and $\mathbf{1 0}$ ported. The absolute stereochemistry of other products $\mathbf{8}$ was presented by analogy.

\section{Conclusion}

The combination of a chiral amidophosphane, chlorobis(ethylene)rhodium dimer, and potassium hydroxide was proven to be a good chiral catalyst for the asymmetric conjugate arylation of acyclic enones with arylboronic acid in a 10:1 mixture of 1,4-dioxane and water. It is important to note that not only cyclic enones of $s$-trans conformation but also acyclic enones of possible $s$-cis conformation are the substrate for the present catalytic asymmetric conjugate arylation with arylboronic acids, although the enantioselectivity is not so high. Further studies towards higher enantioselectivity are the current focus of our efforts.

\section{Experimental}

General Procedure. (R)-4-Phenylnonan-2-one (8aa) ${ }^{12)}$ (Table 1, Entry 8) 1,4-Dioxane $(2.5 \mathrm{ml}), 4 \mathrm{M}$ aqueous $\mathrm{KOH}(0.25 \mathrm{ml})$, and $7 \mathbf{a}(140 \mathrm{mg}$, $1.0 \mathrm{mmol})$ were added to a mixture of $\left[\mathrm{RhCl}\left(\mathrm{C}_{2} \mathrm{H}_{4}\right)_{2}\right]_{2}(5.8 \mathrm{mg}, 0.015 \mathrm{mmol})$, $1(11.6 \mathrm{mg}, 0.033 \mathrm{mmol})$, and $\mathbf{5 a}(610 \mathrm{mg}, 5.0 \mathrm{mmol})$ under argon. The mixture was stirred at $70{ }^{\circ} \mathrm{C}$ for $20 \mathrm{~h}$. After dilution with EtOAc $(40 \mathrm{ml})$, the mixture was washed with $10 \% \mathrm{NaOH}(10 \mathrm{ml})$ and brine $(20 \mathrm{ml})$, and then dried over $\mathrm{Na}_{2} \mathrm{SO}_{4}$. Concentration and silica gel column chromatography (hexane/AcOEt $=30 / 1)$ gave $8 \mathbf{a a}(90 \%)$ as colorless oil: $[\alpha]_{\mathrm{D}}^{20}-15.1(c=1.1$, $\mathrm{CHCl}_{3}$ ). $81 \%$ ee (HPLC: Daicel Chiralcel OB-H, hexane $/ i-\mathrm{PrOH}=100 / 1$, $0.5 \mathrm{ml} / \mathrm{min}, 254 \mathrm{~nm}$; major $16.7 \mathrm{~min}$, minor $13.4 \mathrm{~min}) .{ }^{1} \mathrm{H}-\mathrm{NMR}(500 \mathrm{MHz}$, $\left.\mathrm{CDCl}_{3}\right) \delta: 0.82(\mathrm{t}, J=7.0 \mathrm{~Hz}, 3 \mathrm{H}), 1.10-1.23(\mathrm{~m}, 6 \mathrm{H}), 1.55-1.63(\mathrm{~m}, 2 \mathrm{H})$, $2.01(\mathrm{~s}, 3 \mathrm{H}), 2.69(\mathrm{dd}, J=7.3,16.2 \mathrm{~Hz}, 1 \mathrm{H}), 2.73(\mathrm{dd}, J=7.6,16.2 \mathrm{~Hz}, 1 \mathrm{H})$, $3.10(\mathrm{~m}, 1 \mathrm{H}), 7.16-7.31(\mathrm{~m}, 5 \mathrm{H}) .{ }^{13} \mathrm{C}-\mathrm{NMR}\left(125 \mathrm{MHz}, \mathrm{CDCl}_{3}\right) \delta: 14.0$, 22.4, 27.0, 30.6, 31.7, 36.4, 41.3, 50.9, 126.2, 127.4, 128.4, 144.6, 208.1. IR (neat) $\mathrm{cm}^{-1}: 1710$. MS $m / z: 218\left(\mathrm{M}^{+}\right)$.

$(R)-4-(3-M e t h o x y p h e n y l) n o n a n-2-o n e ~(8 a b)^{14)}$ (Table 2, Entry 1) Eluent, hexane/AcOEt $=40 / 1$. Colorless oil. $[\alpha]_{\mathrm{D}}^{20}-13.9\left(c=1.05, \mathrm{CHCl}_{3}\right) .76 \%$ ee $(\mathrm{OB}-\mathrm{H}$, hexane $/ \mathrm{i}-\mathrm{PrOH}=100 / 1,0.5 \mathrm{ml} / \mathrm{min}, 254 \mathrm{~nm}$; major $38.1 \mathrm{~min}$, minor $23.4 \mathrm{~min}) .{ }^{1} \mathrm{H}-\mathrm{NMR} \quad \delta: 0.82(\mathrm{t}, J=7.0,3 \mathrm{H}), 1.17-1.24(\mathrm{~m}, 6 \mathrm{H})$, $1.52-1.62(\mathrm{~m}, 2 \mathrm{H}), 2.03(\mathrm{~s}, 3 \mathrm{H}), 2.67(\mathrm{dd}, J=7.0,16.0,1 \mathrm{H}), 2.72(\mathrm{dd}$, $J=7.4,16.0,1 \mathrm{H}), 3.07(\mathrm{~m}, 1 \mathrm{H}), 3.79(\mathrm{~s}, 3 \mathrm{H}), 6.72-6.78(\mathrm{~m}, 3 \mathrm{H}), 7.20(\mathrm{dd}$, $J=8.0,8.0,1 \mathrm{H}) .{ }^{13} \mathrm{C}-\mathrm{NMR} \delta: 13.9,22.4,27.0,30.6,31.7,36.3,41.3,50.8$, $55.1,111.2,113.5,119.9,129.4,146.4,159.7,208.1$. IR (neat): 1716. MS $m / z: 248\left(\mathrm{M}^{+}\right)$.

(R)-4-(4-Phenylphenyl)nonan-2-one (8ae) (Entry 2) Eluent, hexane/ $\mathrm{AcOEt}=50 / 1$. White solid. mp $46-48^{\circ} \mathrm{C}$. $[\alpha]_{\mathrm{D}}^{20}-11.7\left(c=0.99, \mathrm{CHCl}_{3}\right)$. $78 \%$ ee $(\mathrm{AD}-\mathrm{H}$, hexane $/ \mathrm{i}-\mathrm{PrOH}=100 / 1, \quad 0.5 \mathrm{ml} / \mathrm{min}, \quad 254 \mathrm{~nm}$; major $18.7 \mathrm{~min}$, minor $20.6 \mathrm{~min}) .{ }^{1} \mathrm{H}-\mathrm{NMR} \delta: 0.84(\mathrm{t}, J=7.6,3 \mathrm{H}), 1.16-1.25(\mathrm{~m}$, $6 \mathrm{H}), 1.55-1.65(\mathrm{~m}, 2 \mathrm{H}), 2.04$ (s, 3H), 2.72 (dd, $J=7.0,16.2,1 \mathrm{H}), 2.76$ (dd, $J=7.4,16.2,1 \mathrm{H}), 3.16(\mathrm{~m}, 1 \mathrm{H}), 7.24(\mathrm{~d}, J=8.0,2 \mathrm{H}), 7.32(\mathrm{t}, J=7.5,1 \mathrm{H})$,

Table 2. Catalytic Asymmetric Conjugate Arylation of Acyclic Enones 7

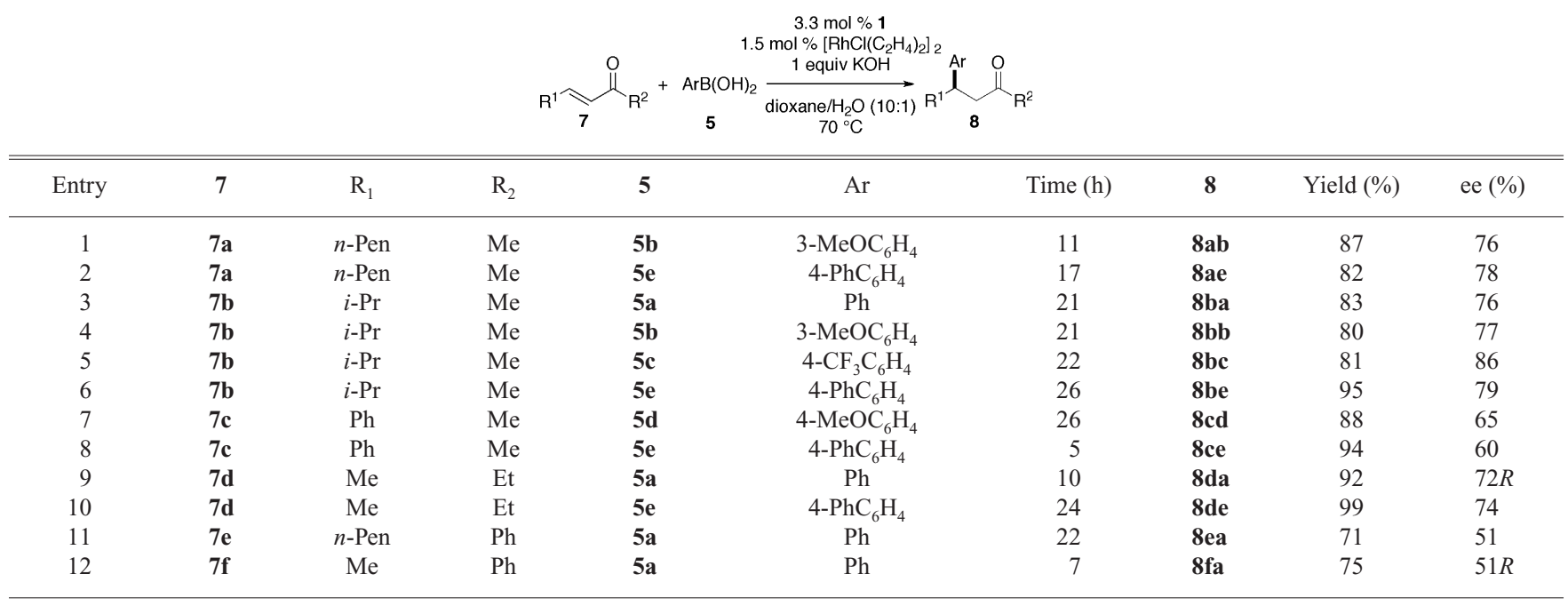


7.42 (dd, $J=7.5,8.0,2 \mathrm{H}), 7.52(\mathrm{~d}, J=8.0,2 \mathrm{H}), 7.57(\mathrm{~d}, J=8.0,2 \mathrm{H}) .{ }^{13} \mathrm{C}-$ NMR $\delta: 13.9,22.4,27.0,30.6,31.7,36.4,40.8,50.8,126.96,127.07$, 127.14, 127.9, 128.7, 139.2, 140.9, 143.8, 208.1. IR (KBr): 1713. MS m/z: $294\left(\mathrm{M}^{+}\right)$. Anal. Calcd for $\mathrm{C}_{21} \mathrm{H}_{26} \mathrm{O}: \mathrm{C}, 85.67 ; \mathrm{H}, 8.90$. Found: $\mathrm{C}, 85.43 ; \mathrm{H}$ 8.99.

(S)-5-Methyl-4-phenylhexan-2-one (8ba) ${ }^{12)}$ (Entry 3) Eluent, hexane $\mathrm{AcOEt}=50 / 1$. Colorless oil. $[\alpha]_{\mathrm{D}}^{20}-25.7\left(c=1.2, \mathrm{CHCl}_{3}\right) .76 \%$ ee $(\mathrm{OD}-\mathrm{H}$, hexane $/ i-\mathrm{PrOH}=50 / 1,0.5 \mathrm{ml} / \mathrm{min}, 254 \mathrm{~nm}$; major $26.4 \mathrm{~min}$, minor $23.1 \mathrm{~min})$ ${ }^{1} \mathrm{H}-\mathrm{NMR} \delta: 0.74(\mathrm{~d}, J=6.7,3 \mathrm{H}), 0.93(\mathrm{~d}, J=6.7,3 \mathrm{H}), 1.83(\mathrm{~m}, 1 \mathrm{H}), 1.98(\mathrm{~s}$, $3 \mathrm{H}), 2.75-2.83(\mathrm{~m}, 2 \mathrm{H}), 2.91(\mathrm{~m}, 1 \mathrm{H}), 7.13-7.20(\mathrm{~m}, 3 \mathrm{H}), 7.25-7.29(\mathrm{~m}$ $2 \mathrm{H}) .{ }^{13} \mathrm{C}-\mathrm{NMR} \delta: 20.2,20.6,30.5,33.2,47.6,48.0,126.3,128.2,128.3$, 143.3, 208.5. IR (neat): 1716. MS m/z: $190\left(\mathrm{M}^{+}\right)$.

$(S)-4-(3-M e t h o x y p h e n y l)-5-m e t h y l h e x a n-2-o n e \quad(8 b b)^{14)} \quad($ Entry 4$)$ Eluent, hexane/AcOEt $=30 / 1$. Colorless oil. $[\alpha]_{\mathrm{D}}^{20}-21.8\left(c=1.16, \mathrm{CHCl}_{3}\right)$. $77 \%$ ee (OD-H, hexane $/ i-\mathrm{PrOH}=100 / 1, \quad 0.5 \mathrm{ml} / \mathrm{min}, 254 \mathrm{~nm}$; major $33.1 \mathrm{~min}$, minor $25.0 \mathrm{~min}$ ). ${ }^{1} \mathrm{H}-\mathrm{NMR} \delta: 0.74$ (d, $\left.J=6.7,3 \mathrm{H}\right), 0.93$ (d, $J=6.7$, $3 \mathrm{H}), 1.82(\mathrm{~m}, 1 \mathrm{H}), 1.99(\mathrm{~s}, 3 \mathrm{H}), 2.75-2.80(\mathrm{~m}, 2 \mathrm{H}), 2.89(\mathrm{~m}, 1 \mathrm{H}), 3.79(\mathrm{~s}$, $3 \mathrm{H}), 6.69-6.75(\mathrm{~m}, 3 \mathrm{H}), 7.19(\mathrm{dd}, J=8.0,8.0,1 \mathrm{H}) .{ }^{13} \mathrm{C}-\mathrm{NMR} \delta: 20.3,20.6$, 30.5, 33.2, 47.6, 48.1, 55.1, 111.2, 114.4, 120.7, 129.1, 145.1, 159.5, 208.4. IR (neat): 1717,1601 . MS $m / z: 220\left(\mathrm{M}^{+}\right)$.

(S)-4-(4-(Trifluoromethyl)phenyl)-5-methylhexan-2-one (8bc) (Entry 5) Eluent, hexane $/ \mathrm{AcOEt}=50 / 1$. Colorless oil. $[\alpha]_{\mathrm{D}}^{20}-9.5 \quad(c=1.12$ $\left.\mathrm{CHCl}_{3}\right) .86 \%$ ee $(\mathrm{AD}-\mathrm{H}$, hexane $/ i-\mathrm{PrOH}=100 / 1,0.5 \mathrm{ml} / \mathrm{min}, 254 \mathrm{~nm}$; major $24.6 \mathrm{~min}$, minor $22.3 \mathrm{~min}$ ). ${ }^{1} \mathrm{H}-\mathrm{NMR} \delta: 0.74$ (d, $\left.J=6.8,3 \mathrm{H}\right), 0.93$ (d, $J=6.4$ $3 \mathrm{H}), 1.84(\mathrm{~m}, 1 \mathrm{H}), 2.01(\mathrm{~s}, 3 \mathrm{H}), 2.80(\mathrm{dd}, J=9.5,16.6,1 \mathrm{H}), 2.86(\mathrm{dd}, J=5.0$, $16.6,1 \mathrm{H}), 3.02(\mathrm{~m}, 1 \mathrm{H}), 7.26(\mathrm{~d}, J=8.0,2 \mathrm{H}), 7.53(\mathrm{~d}, J=8.0,2 \mathrm{H}) .{ }^{13} \mathrm{C}-\mathrm{NMR}$ $\delta: 20.2,20.5,30.4,33.0,47.1,47.5,124.3\left(\mathrm{q},{ }^{1} J_{\mathrm{CF}}=270\right), 125.1 \quad(\mathrm{q}$ $\left.{ }^{3} J_{\mathrm{CF}}=3.1\right), 128.53$ (q, $\left.{ }^{2} J_{\mathrm{CF}}=31.9\right), 128.57,147.8,207.4$. IR (neat): 1717. MS m/z: $258\left(\mathrm{M}^{+}\right)$. Anal. Calcd for $\mathrm{C}_{14} \mathrm{H}_{16} \mathrm{~F}_{3} \mathrm{O}: \mathrm{C}, 65.10 ; \mathrm{H}, 6.63$. Found: $\mathrm{C}$, $64.95 ; \mathrm{H}, 6.63$.

(S)-4-(4-Phenylphenyl)-5-methylhexan-2-one (8be) (Entry 6) Eluent, $\mathrm{C}_{6} \mathrm{H}_{6} / \mathrm{AcOEt}=200 / 1$. White solid. $\mathrm{mp} 66-68^{\circ} \mathrm{C} .[\alpha]_{\mathrm{D}}^{20}-19.9(c=1.07$, $\mathrm{CHCl}_{3}$ ). $79 \%$ ee (OD-H, hexane $/ i-\mathrm{PrOH}=100 / 1,0.5 \mathrm{ml} / \mathrm{min}, 254 \mathrm{~nm}$; major $21.6 \mathrm{~min}$, minor $20.2 \mathrm{~min}$ ). ${ }^{1} \mathrm{H}-\mathrm{NMR} \delta: 0.78$ (d, $\left.J=6.7,3 \mathrm{H}\right), 0.95$ (d, $J=6.7$, $3 \mathrm{H}), 1.86(\mathrm{~m}, 1 \mathrm{H}), 2.01(\mathrm{~s}, 3 \mathrm{H}), 2.82-2.84(\mathrm{~m}, 2 \mathrm{H}), 2.97(\mathrm{~m}, 1 \mathrm{H}), 7.21(\mathrm{~d}$ $J=8.0,2 \mathrm{H}), 7.32(\mathrm{t}, J=7.5,1 \mathrm{H}), 7.42(\mathrm{dd}, J=7.5,8.0,2 \mathrm{H}), 7.50$ (d, $J=8.0$ $2 \mathrm{H}), 7.57(\mathrm{~d}, J=8.0,2 \mathrm{H}) .{ }^{13} \mathrm{C}-\mathrm{NMR} \delta: 20.3,20.6,30.5,33.2,47.5,47.6$, $126.8,126.9,127.1,128.66,128.7,139.1,140.9,142.4,208.3$. IR (neat) 1717. MS $m / z: 266\left(\mathrm{M}^{+}\right)$. Anal. Calcd for $\mathrm{C}_{19} \mathrm{H}_{22} \mathrm{O}: \mathrm{C}, 85.67 ; \mathrm{H}, 8.32$. Found: C, 85.57; H, 8.33

(S)-4-(4-Methoxyphenyl)-4-phenylbutan-2-one $\quad(8 \mathrm{~cd})^{35}$ (Entry $\left.\quad 7\right)$ Eluent, hexane/AcOEt=20/1. Colorless oil. $[\alpha]_{\mathrm{D}}^{20}-0.72\left(c=0.97, \mathrm{CHCl}_{3}\right)$. $65 \%$ ee $(\mathrm{OD}-\mathrm{H}$, hexane $/ i-\mathrm{PrOH}=50 / 1,1.0 \mathrm{ml} / \mathrm{min}, 254 \mathrm{~nm}$; major $37.4 \mathrm{~min}$, minor $44.7 \mathrm{~min}) .{ }^{1} \mathrm{H}-\mathrm{NMR} \delta: 2.07(\mathrm{~s}, 3 \mathrm{H}), 3.14(\mathrm{~d}, J=7.7,2 \mathrm{H}), 3.76(\mathrm{~s}, 3 \mathrm{H})$ $4.54(\mathrm{t}, J=7.7,1 \mathrm{H}), 6.81(\mathrm{~d}, J=8.9,2 \mathrm{H}), 7.12-7.28(\mathrm{~m}, 7 \mathrm{H}) .{ }^{13} \mathrm{C}-\mathrm{NMR} \delta$ $30.6,45.2,49.8,55.1,114.0,126.4,127.6,128.6,128.7,136.0,144.2$, 158.1, 207.1. IR (nujol): 1709, 1605. MS m/z: $254\left(\mathrm{M}^{+}\right)$

(S)-4-(4-Phenylphenyl)-4-phenylbutan-2-one (8ce) (Entry 8) Eluent, $\mathrm{C}_{6} \mathrm{H}_{6} / \mathrm{AcOEt}=100 / 1$. White solid. $\mathrm{mp} 117-119^{\circ} \mathrm{C} .[\alpha]_{\mathrm{D}}^{20}+6.6(c=0.96$, $\left.\mathrm{CHCl}_{3}\right) .60 \%$ ee $(\mathrm{OD}-\mathrm{H}$, hexane $/ i-\mathrm{PrOH}=50 / 1,1.0 \mathrm{ml} / \mathrm{min}, 254 \mathrm{~nm}$; majo $19.6 \mathrm{~min}$, minor $23.3 \mathrm{~min}) .{ }^{1} \mathrm{H}-\mathrm{NMR} \delta: 2.11(\mathrm{~s}, 3 \mathrm{H}), 3.22(\mathrm{~d}, J=7.3,2 \mathrm{H})$, $4.64(\mathrm{t}, J=7.3,1 \mathrm{H}), 7.19(\mathrm{~m}, 1 \mathrm{H}), 7.26-7.33(\mathrm{~m}, 7 \mathrm{H}), 7.41(\mathrm{dd}, J=7.8,7.8$, $2 \mathrm{H}), 7.50(\mathrm{~d}, J=8.0,2 \mathrm{H}), 7.54(\mathrm{~d}, J=7.6,2 \mathrm{H}) .{ }^{13} \mathrm{C}-\mathrm{NMR} \delta: 30.6,45.6,49.6$, $126.5,127.0,127.1,127.3,127.7,128.1,128.6,128.7,139.3,140.8,143.0$ 143.8, 206.8. IR (KBr): 1713. MS m/z: $300\left(\mathrm{M}^{+}\right)$. EI-MS $\mathrm{m} / \mathrm{z}: 300.1504$ (Calcd for $\mathrm{C}_{22} \mathrm{H}_{20} \mathrm{O}: 300.1514$ ).

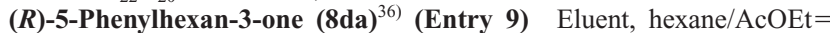
30/1. Colorless oil. $[\alpha]_{\mathrm{D}}^{22}-64.2\left(c=1.3, \mathrm{C}_{6} \mathrm{H}_{6}\right)$; lit. $[\alpha]_{\mathrm{D}}^{22}-56.4(c=1.0$, $\left.\mathrm{C}_{6} \mathrm{H}_{6}\right)$ for $R .72 \%$ ee $(\mathrm{AD}-\mathrm{H}$, hexane $/ i-\mathrm{PrOH}=100 / 1,0.5 \mathrm{ml} / \mathrm{min}, 254 \mathrm{~nm}$; major $11.8 \mathrm{~min}$, minor $10.6 \mathrm{~min}){ }^{1} \mathrm{H}-\mathrm{NMR} \delta: 0.98(\mathrm{t}, J=7.4,3 \mathrm{H}), 1.26(\mathrm{~d}$ $J=7.0,3 \mathrm{H}), 2.26-2.38(\mathrm{~m}, 2 \mathrm{H}), 2.63(\mathrm{dd}, J=8.0,16.2,1 \mathrm{H}), 2.72(\mathrm{dd}$ $J=6.7,16.2,1 \mathrm{H}), 3.32(\mathrm{~m}, 1 \mathrm{H}), 7.17-7.30(\mathrm{~m}, 5 \mathrm{H}) .{ }^{13} \mathrm{C}-\mathrm{NMR} \delta \cdot 7.5,21.8$ $35.4,36.6,50.7,126.3,126.8,128.5,146.3,210.5$. IR (neat): 1713 . MS $m / z$ : $176\left(\mathrm{M}^{+}\right)$.

(R)-5-(4-Phenylphenyl)hexan-3-one (8de) (Entry 10) Eluent, hexane/ $\mathrm{AcOEt}=20 / 1$. White solid. $\mathrm{mp} 54-56^{\circ} \mathrm{C}$. $[\alpha]_{\mathrm{D}}^{20}-36.6\left(c=1.0, \mathrm{CHCl}_{3}\right)$. $74 \%$ ee $(\mathrm{AD}-\mathrm{H}$, hexane $/ \mathrm{i}-\mathrm{PrOH}=100 / 1, \quad 0.5 \mathrm{ml} / \mathrm{min}, \quad 254 \mathrm{~nm}$; major $24.0 \mathrm{~min}$, minor $18.7 \mathrm{~min}$ ). ${ }^{1} \mathrm{H}-\mathrm{NMR} \delta: 1.00$ (t, $\left.J=7.3,3 \mathrm{H}\right), 1.30$ (d, $J=6.7$, $3 \mathrm{H}), 2.29-2.40(\mathrm{~m}, 2 \mathrm{H}), 2.67(\mathrm{dd}, J=8.0,16.2,1 \mathrm{H}), 2.76(\mathrm{dd}, J=6.7,16.2$, $1 \mathrm{H}), 3.37(\mathrm{~m}, 1 \mathrm{H}), 7.28(\mathrm{~d}, J=8.0,2 \mathrm{H}), 7.33(\mathrm{t}, J=7.5,1 \mathrm{H}), 7.42(\mathrm{dd}$ $J=7.5,8.0,2 \mathrm{H}), 7.52(\mathrm{~d}, J=8.0,2 \mathrm{H}), 7.57(\mathrm{~d}, J=8.0,2 \mathrm{H}) .{ }^{13} \mathrm{C}-\mathrm{NMR} \delta: 7.5$, $21.9,35.0,36.6,50.7,127.0,127.1,127.19,127.21,128.7,139.2,140.9$, 145.5, 210.4. IR (neat): 1713. MS $m / z: 252\left(\mathrm{M}^{+}\right)$. Anal. Calcd for $\mathrm{C}_{18} \mathrm{H}_{20} \mathrm{O}$ C, $85.67 ; \mathrm{H}, 7.99$. Found: C, 85.48; H, 8.00.
(R)-1,3-Diphenyloctan-1-one $(8 \mathrm{ea})^{37)} \quad($ Entry 11$)$ Eluent, hexane/ AcOEt $=20 / 1$. White solid. mp $63-64{ }^{\circ} \mathrm{C} .[\alpha]_{\mathrm{D}}^{20}+1.3\left(c=1.1, \mathrm{CHCl}_{3}\right) .51 \%$ ee $(\mathrm{AD}$, hexane $/ i-\mathrm{PrOH}=100 / 1,0.5 \mathrm{ml} / \mathrm{min}, 254 \mathrm{~nm}$; major $19.1 \mathrm{~min}$, minor $15.0 \mathrm{~min}) .{ }^{1} \mathrm{H}-\mathrm{NMR}: 0.82(\mathrm{t}, J=6.4,3 \mathrm{H}), 1.19-1.26(\mathrm{~m}, 6 \mathrm{H}), 1.60-1.72$ $(\mathrm{m}, 2 \mathrm{H}), 3.20-3.35(\mathrm{~m}, 3 \mathrm{H}), 7.17(\mathrm{t}, J=7.5,1 \mathrm{H}), 7.22(\mathrm{~d}, J=7.5,2 \mathrm{H}), 7.28$ (dd, $J=7.5,7.5,2 \mathrm{H}), 7.43$ (dd, $J=7.5,7.5,2 \mathrm{H}), 7.53(\mathrm{t}, J=7.5,1 \mathrm{H}), 7.89$ (d, $J=7.5,2 \mathrm{H}) .{ }^{13} \mathrm{C}-\mathrm{NMR}: 13.9,22.4,27.1,31.7,36.2,41.1,45.9,126.2,127.6$, $128.0,128.4,128.5,132.9,137.3,145.0,199.3$. IR (neat): 1680 . MS $m / z$ : $280\left(\mathrm{M}^{+}\right)$.

$(R)$-1,3-Diphenylbutan-1-one $(\mathbf{8 f a})^{38)} \quad($ Entry 12$)$ Eluent, hexane/ $\mathrm{AcOEt}=50 / 1$. White solid. mp $50-51{ }^{\circ} \mathrm{C} .[\alpha]_{\mathrm{D}}^{25}-7.8\left(c=2.65, \mathrm{CCl}_{4}\right)$; lit. $[\alpha]_{\mathrm{D}}^{25}-14.6\left(c=1.8, \mathrm{CCl}_{4}\right)$ for $R$. $51 \%$ ee $(\mathrm{AD}$, hexane $/ i-\mathrm{PrOH}=100 / 1$, $0.5 \mathrm{ml} / \mathrm{min}, 254 \mathrm{~nm}$; major $10.7 \mathrm{~min}$, minor $8.6 \mathrm{~min}$ ). ${ }^{1} \mathrm{H}-\mathrm{NMR}$ : 1.33 (d, $J=7.0,3 \mathrm{H}), 3.19(\mathrm{dd}, J=8.3,16.5,1 \mathrm{H}), 3.30(\mathrm{dd}, J=5.5,16.5,1 \mathrm{H}), 3.50(\mathrm{~m}$, $1 \mathrm{H}), 7.20(\mathrm{~m}, 1 \mathrm{H}), 7.26-7.32(\mathrm{~m}, 4 \mathrm{H}), 7.44(\mathrm{dd}, J=7.5,7.5,2 \mathrm{H}), 7.55(\mathrm{t}$, $J=7.5,1 \mathrm{H}), 7.92(\mathrm{~d}, J=7.5,2 \mathrm{H}) .{ }^{13} \mathrm{C}-\mathrm{NMR}: 21.8,35.5,47.0,126.3,126.9$, $128.1,128.56,128.59,133.0,137.2,146.6,199.2$. IR (neat): 1682. MS $m / z$ : $224\left(\mathrm{M}^{+}\right)$

Acknowledgments This research was partially supported by a Grant-inAid for Scientific Research, a Grant-in-Aid for Young Scientists (B), a Grant-in-Aid for Scientific Research on Priority Areas "Advanced Molecular Transformations," and Targeted Proteins Research Program from the Ministry of Education, Culture, Sports, Science and Technology of Japan.

\section{References and Notes}

1) Tomioka K., Synthesis, 1990, 541—549 (1990)

2) Kanai M., Shibasaki M., "Catalytic Asymmetric Synthesis," ed. by Ojima I., VCH, Weinheim, 2000, p. 569.

3) Krause N., Hoffmann-Röder A., Synthesis, 2001, 171-196 (2001).

4) Feringa B. L., Naasz R., Imbos R., Arnold L. A., "Modern Organocopper Chemistry," Chapter 7, ed. by Krause N., Wiley-VCH, Weinheim, 2002.

5) Christoffers J., Koripelly G., Rosiak A., Rössle M., Synthesis, 2007, 1279-1300 (2007)

6) Tomioka K., "Comprehensive Asymmetric Catalysis Supplement to Chapter 31.1," ed. by Jacobsen E. N., Pfaltz A., Yamamoto H., Springer, New York, 2004, pp. 109-124.

7) Alexakis A., Benhaim C., Eur. J. Org. Chem., 2002, 3221-3236 (2002).

8) Alexakis A., Bckvall J. E., Krause N., Pmies O., Diguez M., Chem. Rev., 108, 2796-2823 (2008).

9) Harutyunyan S. R., den Hartog T., Geurts K., Minnaard A. J., Feringa B. L., Chem. Rev., 108, 2824-2852 (2008).

10) Our Recent Example: Matsumoto Y., Yamada K., Tomioka K., J. Org. Chem., 73, 4578-4581 (2008).

11) Review: Hayashi T., Yamasaki K., Chem. Rev., 103, 2829-2844 (2003).

12) Takaya Y., Ogasawara M., Hayashi T., Sakai M., Miyaura N., J. Am. Chem. Soc., 120, 5579-5580 (1998).

13) Boiteau J.-G., Imbos R., Minnaard A. J., Feringa B. L., Org. Lett., 5, $681-684$ (2003).

14) Itooka R., Iguchi Y., Miyaura N., J. Org. Chem., 68, 6000-6004 (2003).

15) Shintani R., Tokunaga N., Doi H., Hayashi T., J. Am. Chem. Soc., 126, $6240-6241$ (2004)

16) Shintani R., Duan W.-L., Nagano T., Okada A., Hayashi T., Angew. Chem., Int. Ed., 44, 4611-4614 (2005).

17) Imamoto T., Sugita K., Yoshida K., J. Am. Chem. Soc., 127, 11934 11935 (2005).

18) Okamoto K., Hayashi T., Rawal V. H., Org. Lett., 10, 4387-4389 (2008).

19) Kanai M., Koga K., Tomioka K., Tetrahedron Lett., 33, 7193-7196 (1992).

20) Kanai M., Tomioka K., Tetrahedron Lett., 36, 4273 - 4274 (1995).

21) Kanai M., Nakagawa Y., Tomioka K., Tetrahedron, 55, 3843-3854 (1999).

22) Mori T., Kosaka K., Nakagawa Y., Nagaoka Y., Tomioka K., Tetrahedron: Asymmetry, 9, 3175-3178 (1998).

23) Soeta T., Kuriyama M., Tomioka K., J. Org. Chem., 70, 297-300 (2005).

24) Soeta T., Selim K., Kuriyama M., Tomioka K., Tetrahedron, 63, 6573-6576 (2007)

25) Soeta T., Selim K., Kuriyama M., Tomioka K., Adv. Synth. Catal., 349, 


$$
629-635 \text { (2007). }
$$

26) Selim K., Soeta T., Yamada K., Tomioka K., Chem. Asian J., 3, 342350 (2008).

27) Kuriyama M., Tomioka K., Tetrahedron Lett., 42, 921—923 (2001).

28) Kuriyama M., Nagai K., Yamada K., Miwa Y., Taga T., Tomioka K., J. Am. Chem. Soc., 124, 8932-8939 (2002).

29) Chen Q., Kuriyama M., Soeta T., Hao X., Yamada K., Tomioka K., Org. Lett., 7, 4439-4441 (2005).

30) Chen Q., Soeta T., Kuriyama K., Yamada K., Tomioka K., Adv. Synth. Catal., 348, 2604-2608 (2006).

31) Srgel S., Tokunaga N., Sasaki K., Okamoto K., Hayashi T., Org. Lett., 10, 589-592 (2008).
32) Sakuma S., Sakai M., Itooka R., Miyaura N., J. Org. Chem., 65, $5951-5955$ (2000).

33) The first "a" and second "a" of $8 \mathbf{a a}$ are derived from the suffixes of $\mathbf{7 a}$ and $\mathbf{5 a}$, respectively.

34) Kuriyama M., Soeta T., Hao X., Chen Q., Tomioka K., J. Am. Chem. Soc., 126, 8128-8129 (2004).

35) Defieber C., Paquin J. F., Serna S., Carreira E. M., Org. Lett., 6, 3873 - 3876 (2004).

36) Hubertus A., Horst S., Chem. Ber., 123, 829-836 (1990).

37) Nudelman N. S., Garcia G. V., J. Org. Chem., 66, 1387-1394 (2001).

38) Leitereg T. J., Cram D. J., J. Am. Chem. Soc., 90, 4011- 4018 (1968). 